\title{
Norois
}

Environnement, aménagement, société

\section{Ressources marines, pêche et développement en Argentine : d'une activité de rente au développement des territoires}

Sea resources, fishing and development: from rent seeking to territorial development

\section{Yvanne Bouvet et Claudia Carut}

\section{OpenEdition \\ Journals}

Édition électronique

URL : https://journals.openedition.org/norois/7300

DOI : 10.4000/norois.7300

ISBN : 978-2-7535-7873-9

ISSN : 1760-8546

Éditeur

Presses universitaires de Rennes

\section{Édition imprimée}

Date de publication : 19 juin 2019

Pagination : 65-79

ISBN : 978-2-7535-7871-5

ISSN : 0029-182X

Référence électronique

Yvanne Bouvet et Claudia Carut, « Ressources marines, pêche et développement en Argentine : d'une activité de rente au développement des territoires », Norois [En ligne], 250 | 2019, mis en ligne le 01 janvier 2022, consulté le 13 janvier 2022. URL : http://journals.openedition.org/norois/7300 ; DOI : https://doi.org/10.4000/norois.7300 
La mer épicontinentale de l'Argentine embrasse le plateau continental qui prolonge en Atlantique le plateau patagon et la plaine de la Pampa. Elle est riche de ressources naturelles, sur ses fonds, dans son sous-sol et dans ses eaux où vivent nombre de poissons, crustacés, mollusques et autres céphalopodes ${ }^{1}$. Cet espace maritime apparait stratégique pour l'Argentine qui demeure une économie extravertie et inscrit la pêche comme une activité maritime majeure. Elle est actrice de la politique de développement territorial, notamment dans le sud du pays, en Patagonie et en Terre de Feu.

Cet article est issu d'une collaboration entre l'Université de Brest et l'Universidad Nacional de La Plata (Argentina), se basant sur une analyse bibliographique et une enquête de terrain auprès des acteurs de la filière. L'analyse des documents officiels (discours, rapports et statistiques) portés par l'État argentin, à travers notamment le ministère de la Pêche (MAGyP, Ministerio de Agricultura, Ganaderia y Pesca de la Nación) et l'Institut National de Recherche et développement de la pêche (INIDEP, Instituto Nacional de Investigación y Desarrollo Pesquero), a permis de cerner les débarquements par espèces et par ports, les types de bateaux et méthodes de pêche utilisés, ainsi que les échanges commerciaux. En les confrontant aux entretiens menés dans les ports, auprès des pêcheurs, des représentants des armements et des organismes officiels, nous avons construit l'analyse proposée dans cet article. Dans un contexte de mondialisation des échanges de ressources alimentaires, nous proposons une réflexion sur la place de la pêche en Argentine et les conséquences de l'utilisation des ressources marines dans une logique économique de rente.

Dans une première partie, nous présenterons comment s'est construite la filière halieutique argentine, puis nous nous intéresserons à la mise en place de la stratégie maritime argentine lié à un contexte économique et social fragile, autour de lois permettant l'encadrement de la pêche et la gestion des ressources. Enfin, nous montrerons que depuis la crise de 2001, les évolutions économiques, juridiques et politiques font apparaître plus de concurrences que de complémentarités entre les territoires autour de l'activité halieutique.

1. Nous utiliserons généralement le terme «poisson » pour qualifier l'ensemble des produits de la mer, sauf précision spécifique.

\section{UNE MER AUX RICHESSES MARINES TRÈS EXPLOITÉES ET TRÈS CONVOITÉES}

\section{El Mar Argentino et une ZEE étendue}

El Mar Argentino s'inscrit dans la marge continentale de l'Atlantique sud-ouest, exposée aux effets du courant chaud du Brésil et du courant froid des Malouines, enrichies des apports continentaux fournis par le Rio de la Plata (voir figure 1). Cette mer cache une vaste plateforme continentale étendue depuis l'embouchure du Río de la Plata jusqu'au sud de l'archipel de la Terre de Feu; son extension en longitude est de $2300 \mathrm{~km}$, et varie en latitude de $180 \mathrm{~km}$ au large de la Péninsule Mitre (Terre de Feu) à $880 \mathrm{~km}$ au nord des îles Malouines. Les différences de températures, associées aux mélanges des eaux douces et salées, créent des milieux riches en nutriments, alimentant une diversité de ressources marines à tous les étages, que ce soit en espèces benthiques, démersales ou pélagiques largement exploitées par la pêche (Piola et Rivas, 1997, p. 123). On peut distinguer deux grands espaces maritimes, el Mar Patagonica, au sud du Rio Negro, dominée par les eaux froides, et el Mar Bonaerense, où les eaux subtropicales se chargent de sédiments issus du Rio de la Plata.

Cette plateforme présente une grande importance économique et politique, car en plus des ressources marines vivantes, elle renferme des réserves d'hydrocarbures ainsi que des sables et des graviers. Depuis 2016, l'État argentin a agrandi sa zone économique exclusive (ZEE) à la dimension de la plateforme continentale après 20 ans de tractations avec la Convention des Nations unies sur le droit de la mer (CONVEMAR), faisant jouer le régime de conservation des ressources (Le Bail, 1998). Ainsi la juridiction de l'Argentine porte sur $1785000 \mathrm{~km}^{2}$ de mers, de sols et de sous-sols, y compris les alentours des îles Malouines, les îles de Géorgie du sud et les îles Sandwichs du sud, faisant osciller la limite jusqu'à 369 milles nautiques (COPLA, 2016)2. Cette

\footnotetext{
2. L’Argentine a entériné la Convention de Montego Bay le 31 décembre 1995. En 2009, la République argentine a soumis une demande d'extension de sa ZEE aux limites du plateau continental, conformément au paragraphe 8 de l'article 76 de la Convention. Cette demande, adoptée en mars 2016 par la Commission des limites du plateau continental de l'ONU ne règle pas pour autant la question des îles Falkland et antarctiques. (voir aussi Comisión Nacional del límite exterior de la Plataforma continental Argentina (2016) [http://www.plataformaargentina.gov.ar/]).
} 


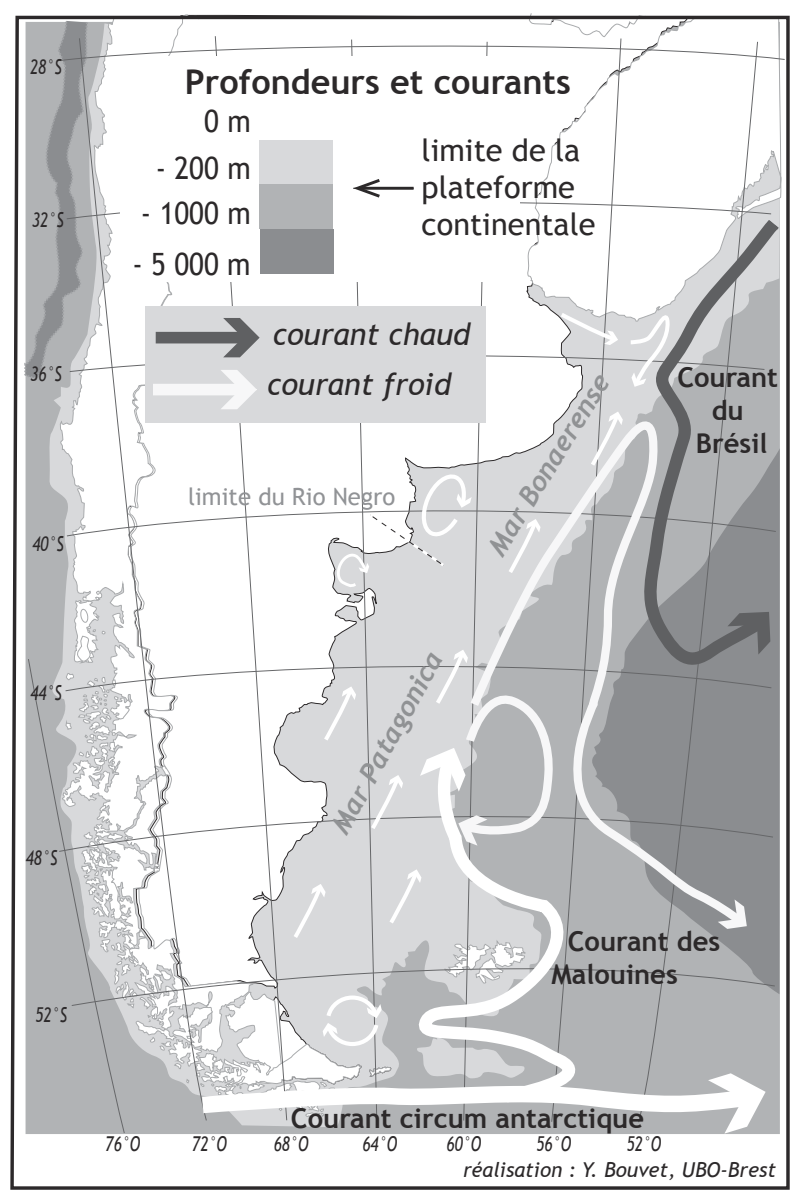

Figure 1 : Plateforme continentale, courants marins et souveraineté de la mer Argentine (sources : Instituto geográfico militar, Campagna, C. et al., 2005)

Continental shelf, sea currents and Argentine sea sovereignty

extension de la ZEE conforte la responsabilité de l'Argentine sur l'utilisation des ressources naturelles d'origine marine en Atlantique sud-ouest.

\section{Une activité halieutique récente}

La pêche maritime s'est développée en Argentine dans la deuxième moitié du $\mathrm{xx}^{\mathrm{e}}$ siècle, reposant essentiellement sur la production du merlu argentin (Merluccius hubbsi) dans les années 1970 auquel est venu s'ajouter l'encornet rouge argentin (Illex argentinus), le merlan bleu (Micromesistius australis), le grenadier patagonien (Macruronus magellanicus) dans les années 1990 et la crevette (Pleoticus muelleri) depuis les années 2000. Malgré cette diversification, la production halieutique argentine continue de reposer sur un nombre limité d'espèces.
L'Argentine s'est tournée tardivement vers l'exploitation des ressources marines (voir figure 2), avec des phases d'expansion liées à des investissements, souvent étrangers, dans l'outil de production, intégrant des navires mieux équipés, plus productifs dans la flottille halieutique. Les années 1970 voient arriver des grands navires de pêche fraîche (chalutiers), puis des navires congélateurs entraînant le développement de la pêche hauturière. La décennie suivante connait le développement des nouvelles pêcheries (crevettes et calamar) et les années 1990 sont celles de l'ouverture néolibérale de l'économie argentine, qui entraîne une forte exploitation des ressources marines conduisant à une production de type minier (Coccaro et al., 2001, p. 35) atteignant presque 1,4 million de tonnes en 1997, avant l'effondrement des années 2000. Depuis, dans un contexte de baisse constante, hormis un sursaut en 2006 où le million de tonnes était à nouveau atteint, l'activité halieutique argentine, avec 776000 tonnes en 2017, se concentre sur quelques espèces, et s'exerce à partir des ports de pêche principaux comme Mar del Plata, Puerto Madryn et Puerto Deseado (voir figure 7).

La production halieutique est dominée par la pêche industrielle et $70 \%$ provient de trois espèces. Le merlu argentin (Merluccius hubbsi) ou merluza, est pêché sur toute la plateforme continentale, autant par des navires de pêche fraîche que par des navires-usines congélateurs; il représente $36 \%$ de la production totale soit 281000 tonnes en 2017. La crevette (Pleoticus muelleri) ou langostino, représente presqu'un tiers des captures totales avec 242000 tonnes; elle est plutôt pêchée par des chalutiers dans le sud de la Mer argentine (avec des chaluts à perche, appelés tangoneros), et débarquée dans les ports de Patagonie, pour les deux tiers par des navires-usines congélateurs et un tiers en pêche frầche.

L'encornet rouge argentin (Illex argentinus) représente $16 \%$ des captures avec moins de 100000 tonnes produites en 2017, captures effectuées par des navires de pêche industrielle, les poteros, utilisant des palangres automatiques et des lamparos pour attirer, la nuit, ces milliers de calamars qui pullulent dans les eaux hauturières de la Mer argentine. Une fois congelés en bloc, ceux-ci sont ensuite débarqués pour plus de $60 \%$ à Mar del Plata, et le reste dans les ports patagons. 


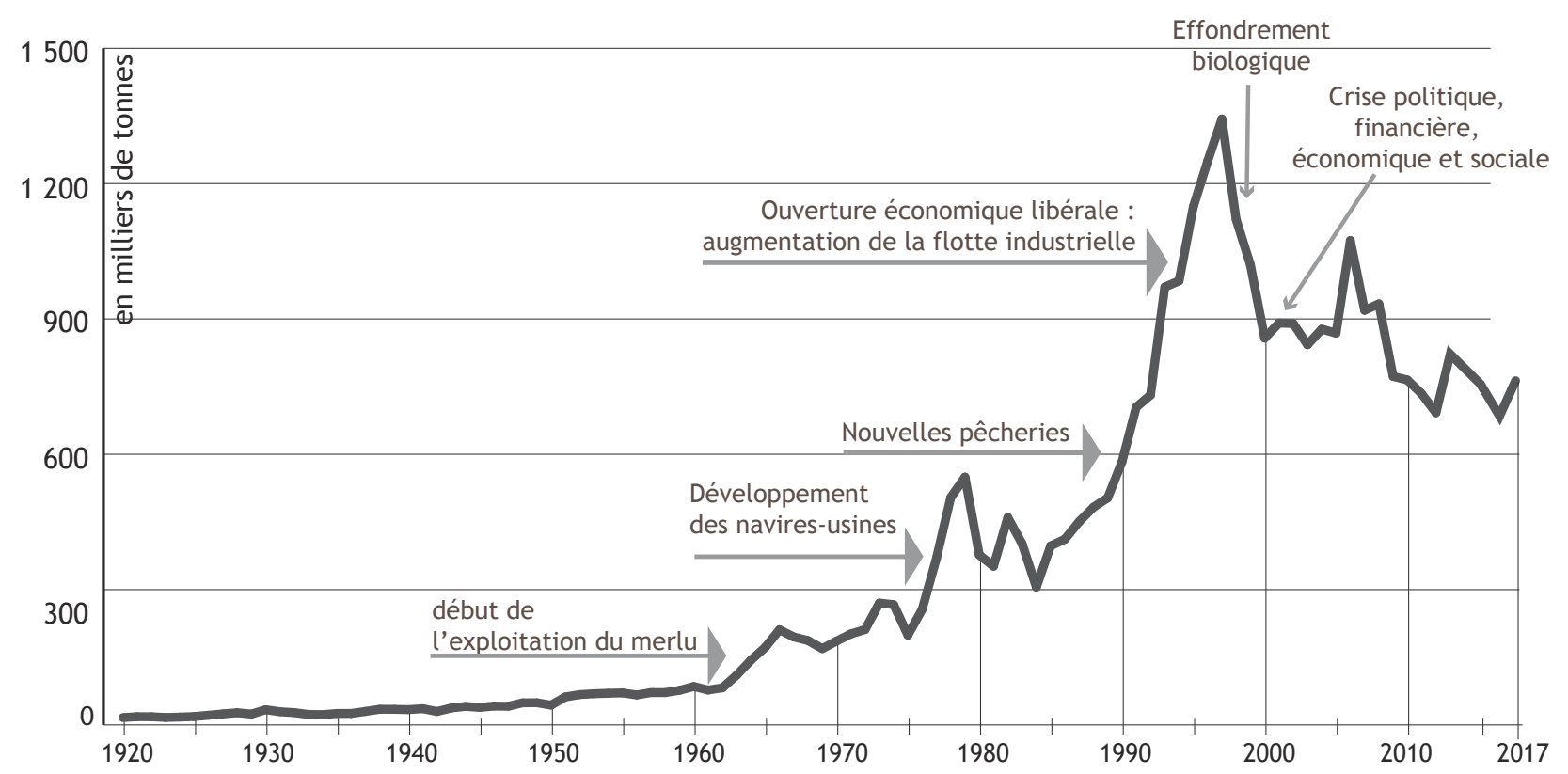

Figure 2 : Évolution des débarquements de la pêche argentine de 1920 à 2017 (source : SAGyP, 2018) Argentina fish landings 1920-2017

La quatrième espèce vient loin derrière les autres puisqu'avec 22000 tonnes produites en 2017, soit $3 \%$ de la production, le grenadier ou merluza de cola (Macruronus magellanicus) est pêché par la flotte industrielle de navires-usines, avant d'être débarqué à Ushuaïa et à Puerto Madryn.

La pêche côtière, et notamment artisanale, a une production plus diversifiée, puisque plus de 70 espèces sont recensées par l'INIDEP, 60 espèces de poissons, cinq de crustacés et cinq de mollusques (Navarro et al., 2014).

Alors que les informations sur les débarquements sont faciles d'accès (voir les sites du ministère de l'Agro-industrie et de l'INIDEP) ${ }^{3}$, les statistiques concernant l'évolution et la répartition de la flottille le sont peu, reposant sur l'immatriculation des navires à la Préfecture navale. La flottille apparait hétérogène, se répartissant entre une flottille industrielle de plus de 600 bateaux (Hobert et al., 2010, p. 20-22) possédant des permis de pêche délivrés par le Conseil Fédéral de la Pêche (CFP), auquel il faut ajouter les 400 bateaux avec des permis de pêche provinciaux. Une répartition selon les lieux de pêche, la taille des bateaux et les méthodes utilisées peut être faite (voir figure 3).

3. [www.agroindustria.gob.ar/sitio/areas/pesca_maritima/;www.inidep.edu. $\mathrm{ar} /]$.
La flotte de pêche de proximité, souvent qualifiée d'artisanale (flota pesquera de pequeña escala) est constituée d'embarcations de moins de 18 mètres, avec un équipage maximal de huit personnes, sans équipement particulier ni de navigation, ni de conservation du poisson. Opérant en rade, dans les fleuves, ou dans la mer territoriale, elle pratique la pêche fraîche sur plusieurs espèces de poissons pélagiques (anchois, maquereau...) ou d'espèces de fonds meubles (crevette, merlu...) avec des méthodes de pêche passives et souvent artisanales (casiers, filets, lignes, senne...). Ces petits bateaux jaune-orange, telles les lanchas amarillas du port de Mar Del Plata, opèrent dans les eaux sous juridiction des Provinces. Cette flottille côtière représente $23 \%$ des unités de la flottille nationale.

Les flottes côtière et hauturière de pêche fraîche (flota costera y buques fresqueros) rassemblent des navires de 18 à 27 mètres qui sont souvent des chalutiers avec une cale réfrigérée pour conserver le poisson entier ou éviscéré. Ces navires partent pour des marées de cinq à quinze jours entre 24 et 100 milles, ramenant le poisson (merlu, lieu), aux usines de traitement et de conditionnement à terre. Cette flottille représente près de $39 \%$ de la flottille nationale. 


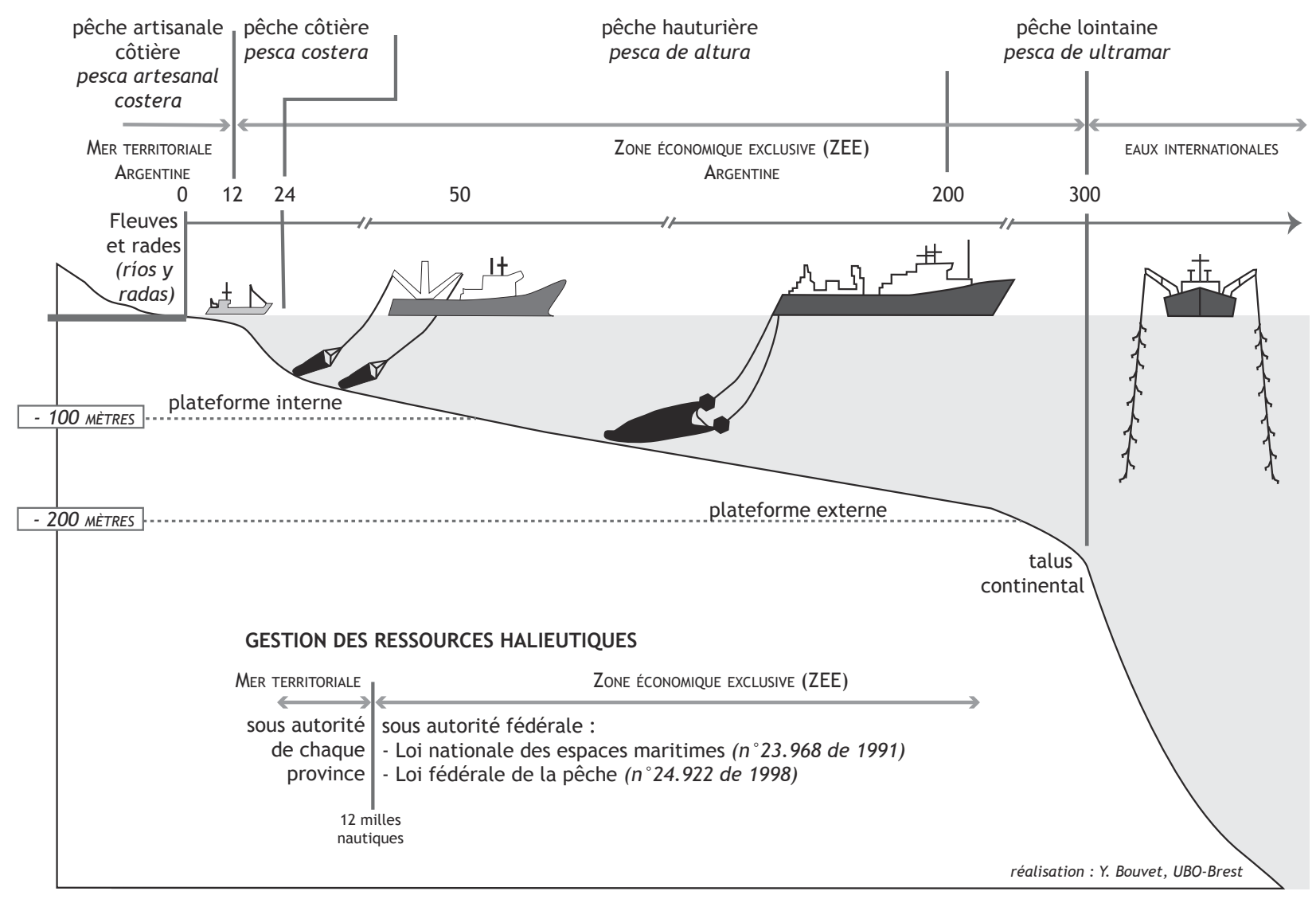

Figure 3 : L'espace halieutique argentin : zones de pêche et juridictions (sources : INIDEP, Observatorio de políticas públicas) Argentina fisheries landind: area and jurisdiction

La flotte hauturière de navires-usines (buques procesadores congeladores) rassemble des navires de grande taille (plus de 25 m jusqu'à $90 \mathrm{~m}$ ) pouvant traquer, pêcher, transformer et congeler le poisson directement à bord. Ces navires sont des chalutiers, de fond pour des espèces comme le merlu (espèce reine des poissons blancs) ou le grenadier, semipélagique pour le merlan bleu (qui servira à faire du surimi), pélagiques pour la crevette, mais aussi des palangriers pour les espèces de grands fonds comme le grenadier, ou des poteros (palangriers et lamparo) pour le calamar. Cette flottille représente près de $38 \%$ de la flottille nationale et opère dans les eaux les plus lointaines de la ZEE argentine, voire dans les eaux internationales.

Une nette séparation existe entre la flottille de pêche fraîche (47 \% de la production halieutique en quantité), qui utilise les usines de transformation installées dans les ports de débarquement et alimentent le marché intérieur, et la flottille des navires-usines (53\% de la production), qui peut expédier le poisson transformé et conditionné à bord directement vers les marchés extérieurs. Sur le graphique suivant, les caractéristiques des flottilles peuvent se voir selon les provinces, avec une production de produits congelés de plus en plus forte au sud du pays, limitant l'activité de transformation à terre (voir figure 4).

L'ensemble de ces flottilles fait travailler directement de 20000 personnes (selon la FAO, 2015) à 40000 personnes selon le Sous-secrétariat à la pêche et à l'aquaculture argentin (Clarin, 28/12/2014) auquel on peut adjoindre un millier de personnes exerçant une activité de pêche artisanale, avec ou sans bateau, parfois sans permis de pêche, pratiquée sur presque tout le littoral argentin (UAPA, 2016) ${ }^{5}$.

\footnotetext{
4. "Con exportaciones récord, el pescado no queda sin vender", Clarin, 28/12/14, [http://www.ieco.clarin.com/economia/exportaciones-recordpescado-queda-vender_0_1274872717.html].

5. Entretien avec Me Corradini, coordinatrice de l'Union argentine des pêcheurs artisans (UAPA), novembre 2016.
} 


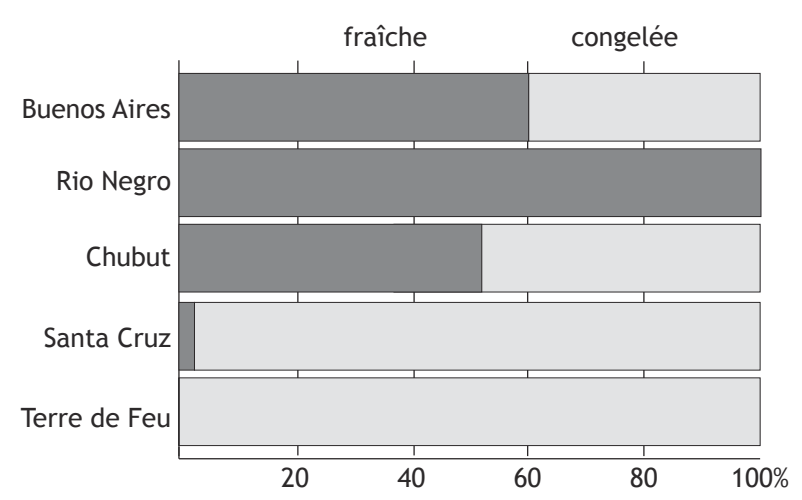

Figure 4 : Répartition de la production selon les flottilles par province en 2017 (source : SAGyP, 2018)

Fish landings by fleet and provinces in 2017

\section{Une filière intégrée aux marchés mondiaux}

L'activité halieutique argentine dépend des marchés internationaux. Plus de la moitié de la production (environ 450000 tonnes en 2016) est exportée. Ces exportations représentent 1725 MUS\$ en 2016 et $2 \%$ de l'ensemble des exportations en valeur de l'Argentine, à un prix moyen assez élevé (3,8 US\$/kg en moyenne en 2016, mais atteignant jusqu'à 6 US\$ pour l'Europe et les États-Unis). Elles concernent une centaine de pays, avec une forte présence de l'Europe (34\% des exportations soit 630 MUS\$, principalement vers l'Espagne et l'Italie), de l'Asie pour un quart des exportations dont la Chine (20\% des exportations en quantité et une valeur de 140 MUS\$) et du Japon (7\% des exportations en quantité pour une valeur de 144 MUS\$). Plus loin derrière, on trouve les pays américains, dont le Brésil (8\% des exportations et 122 MUS\$ en valeur), et les États-Unis (6\% des exportations et 110MUS\$ en valeur). Le marché extérieur se concentre sur les trois espèces principales qui représentent près de $90 \%$ des exportations, les crevettes (160000 tonnes et plus d'un milliard de US\$), suivi du merlu (108000 tonnes et $250 \mathrm{M} \$ \mathrm{US}$ ), et du calamar avec $8 \%$ de la valeur exportée (47000 tonnes et 100 MUS\$).

La variation des exportations depuis 2001 est liée à plusieurs facteurs; le premier est celui de l'évolution interannuelle de la production, suivi de la fluctuation de la demande extérieure et de l'évolution du cours des produits de la mer (voir figure 5). Ainsi, après une année record de production en 2006

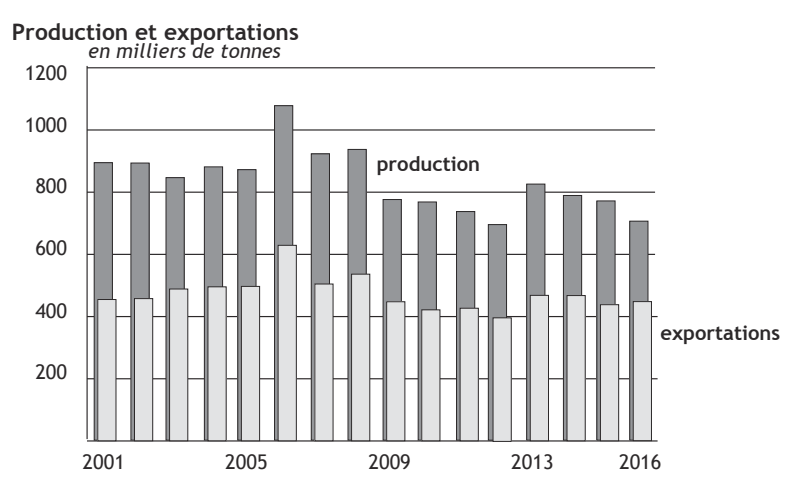

Figure 5 : Production et exportations des produits de la pêche de 2001 à 2016 (source : SAGyP, 2017)

Fish landings and fish products exportations 2001-2016

(628000 tonnes exportées), la quantité des exportations est plutôt à la baisse jusqu'en 2012, en lien avec une chute des prix du calamar et des crevettes, qui rend les marées moins rentables. C'est aussi la conséquence de la moindre productivité du merlu, liée aux fortes captures de la décennie précédente et à l'essoufflement de la ressource (Cañete, 2008, p. 133). Pourtant, la demande du marché extérieur reste forte, notamment en provenance d'Europe, d'Asie du sud-est et surtout de Chine.

Si plus de la moitié de la production halieutique est exportée, le marché intérieur se développe peu à peu, selon les quelques informations que détient le MAGyP et les données de la FAO. Ainsi, la consommation de poisson a particulièrement progressé en Argentine, passant de 5,8 kg par an et par habitant en 2009 , à $9,7 \mathrm{~kg} / \mathrm{an} / \mathrm{habitant}$ en 2014, même si on est encore loin de la moyenne mondiale (19 kg/an/habitant). Cette augmentation est due en partie à des initiatives du gouvernement pour promouvoir la consommation du poisson comme celle de Pescado para todos, (Du poisson pour tous) mis en place de 2010 à 2014 par Cristina Kirchner et repris depuis 2015 par le Movimiento Evita et la CTEP (Confederación de Trabajadores de la Economía Popular); il est constitué de camions frigorifiques partant de Mar del Plata pour aller vendre à tarifs préférentiels du poisson à l'intérieur du pays, et toucher les consommateurs modestes, éloignés des lieux d'approvisonnement. Il fut suivi par un programme Promoción Interna de los Recursos Vivos del Mar (Promotion interne des ressources vivantes de la mer) porté depuis 2012 par le MAGyP, qui vise à stabiliser les 
prix du poisson sur plusieurs mois alors que dans le même temps ceux de la viande bovine ou du poulet augmentent. Ce programme est expérimenté dans cinq provinces (La Pampa, Río Negro, Neuquén, Mendoza, Córdoba) et 96 villes de l'intérieur de l'Argentine. Le but est de proposer des poissons frais peu chers pour modifier les habitudes alimentaires des Argentins (encore très consommateurs de viande bovine, $65 \mathrm{~kg} / \mathrm{an} / \mathrm{h}$ abitant et de volaille, $38 \mathrm{~kg} / \mathrm{an} / \mathrm{habitant}$ ) et de mieux valoriser les productions des pêcheurs côtiers. Pourtant, c'est le merlu qui reste la base de la consomation intérieure, en frais ou congelé, suivi par le calamar (Sesar, 2015, p. 28). Les entreprises de production et de transformation de produits de la mer ont également construit des circuits de distribution avec les supermarchés comme La Anónima, Carrefour ou Jumbo pour vendre les produits congelés sur le marché argentin, soit sous leurs propres marques (Artico, Weggy), soit sous les marques de distributeurs.

Malgré la diversité de sa production, l'Argentine importe aussi pour 47800 tonnes de produits marins, pour un coût de près de 183MUS\$, essentiellement des conserves (75\%), et aussi des produits frais (12\%), en provenance d'Équateur (35\%), du Chili (30\%), de Thaïlande (26\%) et du Brésil $(5 \%)^{6}$.

Au vu des éléments présentés, l'Argentine apparait comme un pays halieutique, proposant une filière organisée de production, de transformation et de commercialisation d'une ressource marine abondante et diversifiée. Cependant, la concurrence est rude entre d'un côté exporter et faire entrer des devises, et de l'autre construire un marché intérieur. Face à la pression exercée par la dette, il est difficile de résister à un prix deux à trois fois plus important à l'extérieur plutôt que de s'appuyer sur un marché intérieur fragile, soumis à une baisse des revenus de la population, renforcée par une inflation galopante (40\% officielle en 2016). Les ressources halieutiques apparaissent comme une manne et la pêche industrielle est l'un des outils de l'économie de rente d'exportation de l'État argentin. Pourtant, la production reste fragile car la gestion des pêcheries est encore récente.

6. [www.agroindustria.gob.ar].

\section{Base Législative, CONTEXTE ÉCONOMIQUE ET POLITIQUE POUR CONSTRUIRE UNE STRATÉGIE HALIEUTIQUE ARGENTINE}

\section{Une gestion fédérale des espaces maritimes}

L’Argentine est une République Fédérale et la gestion de son espace maritime relève de plusieurs autorités, selon la distance au trait de côte (ou ligne de base) avec comme référence est la Ley sobre les espacios marítimos argentinos $n^{\circ} 23.968$ de diciembre $1991^{7}$. La mer territoriale - les eaux intérieures et les espaces en deçà des 12 milles - sont gérées par les États provinciaux; au-delà et jusqu'à la limite des eaux internationales, les eaux sont de la compétence de l'État Fédéral (voir figure 3). La Zone commune de Pêche (ZCP), est gérée avec l'Uruguay par la Comisión mixta del Frente Maritimo, créée par le Traité du Río de La Plata dans les années 1960 (voir figure 6). Le différend de souveraineté des Malouines est toujours en négociation auprès de la CONVEMAR et deux zones sont identifiées par les autorités des îles Malouines, la Falkland Islands Interim Conservation and Management Zone (FICZ) qui représente une zone de 50 milles nautiques autour des îles, et la Falkland Islands Outer Conservation Zone (FOCZ) qui l'étend à l'est et au sud de la précédente (Falkland Islands Government, 2015, p. 26).

Au début des années 1990, pour trouver des devises et soutenir le programme politique libéral du Président Menem, l'État argentin choisit d'exploiter les ressources halieutiques pour les exporter. Cette volonté de vendre à l'extérieur les produits marins coïncide avec le besoin des Européens d'approvisionner leur marché, et de reconvertir leurs flottilles de pêche vers d'autres pêcheries, au moment où se mettent en place les mesures de gestion des ressources des eaux européennes. Un accord est conclu avec l'Europe, accordant des droits de pêche aux flottilles européennes, avec la possibilité de créer des entreprises conjointes (Argentine/Europe), permettant le transfert de flottilles, en échange d'une ouverture du marché européen. Si la production et les exportations augmentent, c'est une catastrophe pour la ressource entraînant une chute de la pro-

7. [www.opds.gba.gov.ar]. 


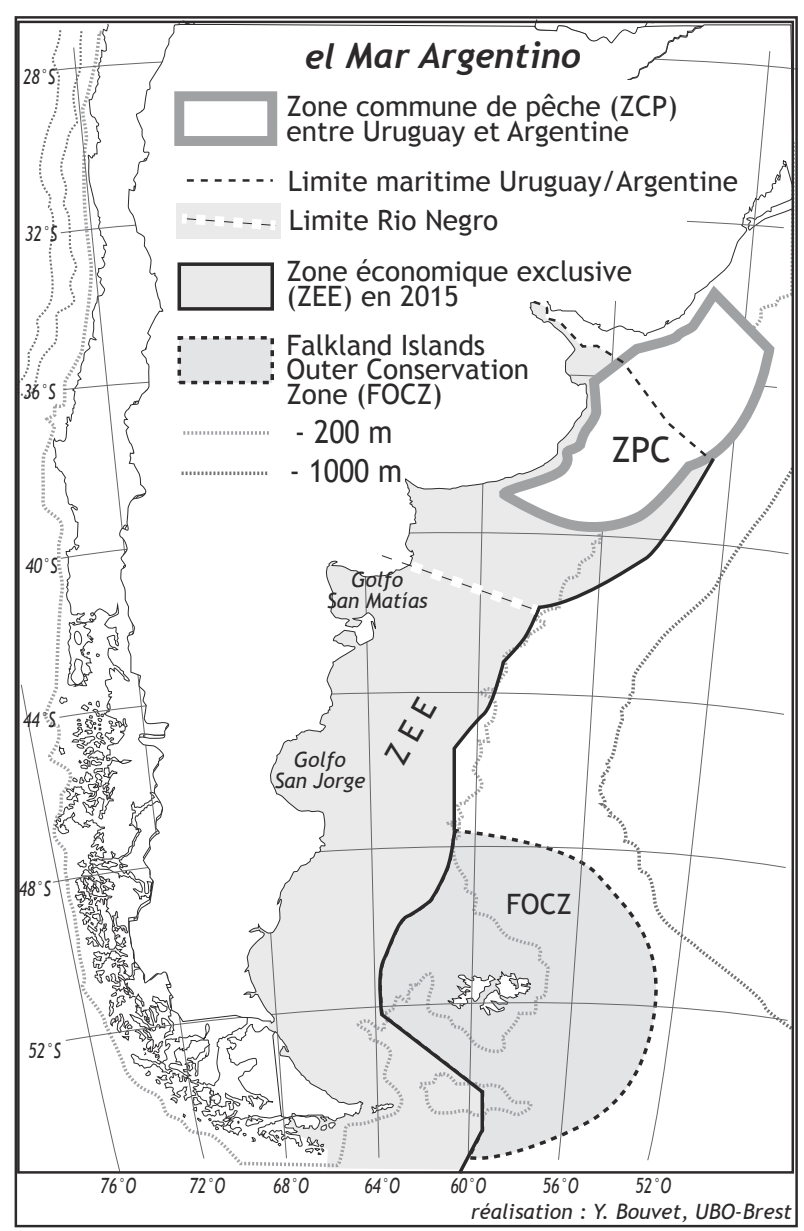

Figure 6 : Délimitation de la souveraineté de la mer Argentine (sources : Instituto geográfico militar, COPLA, 2016)

Limits of state sovereignty in Argentine sea

duction à partir de 1996. Il y a peu de mesures de protection, chaque armateur pêchant un maximum de poissons avant la fermeture des pêcheries, créant des périodes de forte activité des flottilles et d'autres d'inactivité, déstabilisant la pêche mais aussi l'activité de transformation, générant du chômage pour les pêcheurs et les ouvriers d'usines.

Une première réflexion est alors entreprise pour réguler et gérer les pêcheries en Argentine, précédée en 1995 par l'adjonction de l'article 41 à la Constitution nationale qui porte notamment le droit à un environnement sain, équilibré, à une utilisation rationnelle des ressources et à la préservation de la diversité biologique ${ }^{8}$. C'est la fin du sistema olympico où sévissait la loi du plus gros, du plus rapide, du plus habile. Sous les pressions sociales et politiques, ce système olympique va être remplacé par une loi

8. Voir l'intégralité de l'article sur : [www.opds.gba.gov.ar]. pour accompagner le secteur halieutique argentin, qui va dénoncer les accords avec l'Europe dès 1999 (Hobert et al., 2010).

\section{Une loi pour la pêche}

La Ley Federal de Pesca ${ }^{9}$ devient la référence en matière de pêche maritime en Argentine. Cette loi affirme le rôle essentiel du Consejo Federal Pesquero, (CFP), qui est désigné comme l'organe chargé d'établir une politique halieutique nationale. La loi envisage les divers aspects de la pêche maritime, la recherche, les accords internationaux, la préservation et la gestion des ressources à travers une mesure essentielle, l'attribution des quotas individuels transférables (cuotas individuales transferibles de captura, CITC).

Elle est complétée par la Ley General del Ambiente $n^{\circ} 25.675$ (Loi générale sur l'environnement), promulguée en 2002, qui vient apporter d'autres éléments pour préserver et gérer les ressources naturelles, et prône l'amélioration de la qualité de vie des populations, et porte un développement durable, (écologique, économique, social) par la participation des acteurs locaux dans les décisions. Pourtant, cette loi n'est que rarement citée par les acteurs de la pêche, que ce soit dans les instituts de recherche ou les chambres syndicales ou d'entreprises.

Plusieurs instances interviennent aussi dans la construction de la politique et des actions du secteur halieutique. Sous l'autorité de Ministerio de Agroindustria, le sous-secrétariat à la pêche et à l'aquaculture (SAGPyA) porte le discours et les actions gouvernementales et gère deux organismes décentralisés, l'Institut de recherche et développement à la pêche (INIDEP) et le Service national de santé et de qualité agroalimentaire (SENASA). L'INIDEP a pour mission de concevoir des programmes de recherche sur les pêcheries, les techniques d'aquaculture, les méthodes de pêche et l'économie de la pêche, en promouvant un usage rationnel de ressources pour préserver l'écosystème marin. L'INIDEP a aussi un rôle de conseil auprès du SAGPyA, du CFP (Bertolotti, 2008, p. 4). Le SENASA assure le contrôle et la qualité des produits d'origine marine débarqués avant la commercialisation, que ce soit vers le marché intérieur ou vers

9. Ley n 24.922, négociée en 1997 et publiée en 1999 (Decreto $\left.\mathrm{n}^{\circ} 748 / 1999\right)$. 
l'étranger. D'autres organismes nationaux influent sur la gestion des pêcheries, comme le ministère des Relations extérieures et du culte, qui négocie les accords commerciaux et politiques avec les États étrangers, le ministère de l'Environnement et du Développement durable, chargé de la protection des ressources naturelles renouvelables, ainsi que tous les États provinciaux argentins ayant un littoral qui ont leurs propres administrations et règlements. Il faut aussi ajouter les institutions entrepreneuriales, regroupées au sein de chambres nationales, provinciales, représentantes d'intérêts variées tant d'entreprises que de syndicats de pêcheurs ou d'ouvriers.

Le Conseil fédéral de la pêche est composé de représentants désignés (et non élus), placés sous l'autorité du sous-secrétaire d'état à la pêche; on y trouve un représentant du secrétariat d'État aux ressources naturelles et au développement durable, un représentant du ministère des relations extérieures, deux représentants de l'État, et un représentant de chaque province maritime (quelle que soit l'importance de l'activité de pêche dans la province). Le CFP est doublé d'une commission technique (comisión asesora honoraria) qui rassemble les représentants des syndicats d'entreprises et de travailleurs du secteur halieutique, sans que le mode de désignation et de participation soit explicité. Pourtant, la composition du CFP et de la commission technique est fondamentale dans la construction de la politique halieutique de l'Argentine, puisque la stratégie halieutique s'y construit. Et tous les représentants des syndicats ou des entreprises n'ont pas le même pouvoir à l'intérieur de la commission voire du conseil, notamment dans l'attribution des quotas de captures ou de financement de la recherche.

\section{Des quotas individuels transférables et de la surveillance}

L'outil privilégié de la politique halieutique argentine est le régime des quotas individuels transférables de capture (CITC) ${ }^{10}$, censé assurer la gestion durable des espèces marines. Fixés par le CFP, les CITC sont attribués par permis de pêche, c'est-àdire par bateau. Chaque quota est défini par zone et mode de capture, par espèce, par saison, en fonction des informations transmises par l'INIDEP sur

10. Article 27 de la loi no 24.922 les quantités maximales admissibles. L'attribution des CITC concernent les armements industriels et se fait selon l'historique de production de chaque bateau (avec une moyenne faite entre 1988 et 1996, période de fortes captures, notamment de merlu), et aussi selon la quantité de main-d'œuvre argentine employée sur les bateaux (au minimum $75 \%$ pour les équipages), tout en prêtant attention aux investissements effectués dans les usines de transformations en Argentine et le nombre d'emplois générés. Tout cela est pondéré par d'éventuels antécédents de sanction pour non-respect des règlements maritimes. L'attribution effective des CITC selon ces critères est difficile à appliquer, et ce sont les grands armements qui s'en sortent le mieux. Les CITC ne peuvent être transférés entre bateaux de pêche fraîche et navires congélateurs, tout comme un pourcentage maximal de CITC ne peut être attribué à une seule entreprise, pour éviter d'attribuer des CITC à des investisseurs étrangers.

L'affirmation du rôle central de l'État dans une gestion commune et encadrée des ressources naturelles marines à travers cette loi, et particulièrement avec l'instauration de quotas, a pris beaucoup de temps. Les âpres discussions entre les représentants de l'État, des Provinces, des nombreuses chambres d'entreprises et des syndicats se sont déroulées dans un contexte de crise politique, économique (fin de la convertibilité peso/dollar), sociale (explosion du chômage et de la pauvreté) et biologique (chute phénoménale de la production de merlu) du début du $\mathrm{XXI}^{\mathrm{e}}$ siècle, ce qui a exacerbé la concurrence et la concentration capitalistique du secteur. Ce n'est finalement qu'en 2010 que furent mis en place les premiers CITC, négociés pour quinze ans. Ces quotas concernent les quatre espèces très demandées sur le marché international (merlu, merlan bleu, grenadier, légine australe dit merluza negra). Ainsi, moins de 250 bateaux ont reçu un CITC, un tiers pour des navires congélateurs, deux tiers pour des bateaux de pêche fraîche.

Le SAGPyA est l'autorité chargée d'appliquer les décisions prises par le CFP, dans les eaux soumises à la compétence nationale, et de faire converger les décisions et lois prises par les Provinces, qui gèrent les eaux intérieures et la mer territoriale. Des moyens de contrôle sont mis en place avec la Préfecture navale argentine, qui vérifie la conformité des navires, des équipages et les déclarations 
de captures. L'INIDEP dispose aussi d'un ensemble d'observateurs embarqués pour connaitre les tailles des espèces, leurs lieux de pêche et le SENASA vérifie et enregistre les débarquements (quantités et qualités) dans chaque port argentin. Le CFP estime que $90 \%$ des bateaux possédant des CITC sont contrôlés, même s'il ne dit pas combien respectent effectivement les quotas.

Si la loi fédérale sur la pêche affirme la nécessité de préserver la ressource naturelle marine, premier pas qui doit être salué, elle ne constitue pas une stratégie, sauf à penser que la répartition d'un bien commun entre diverses entreprises commerciales forme l'unique politique de développement économique, social ou territorial nationale et régionale. Il n'y a pas encore en Argentine de reconnaissance d'une dépendance des communautés littorales à l'activité de pêche, et donc à la protection de la ressource marine, garante de l'activité économique et sociale des communautés atlantiques.

\section{LA PÊCHE EN Argentine ENTRE TENSION POLITIQUE, ÉCONOMIQUE, SOCIALE ET OUTIL DE DÉVELOPPE- MENT DURABLE}

\section{Développement des ports du sud}

Les ports de Patagonie se sont développés avec la mise en œuvre de nouvelles pêcheries de la Mer argentine depuis les années 1980, d'abord autour du merlu, puis des crevettes et du calamar, et avec l'arrivée de nouveaux bateaux et l'implantation d'usines de transformation et d'espaces de stockage frigorifiques (voir figure 7 ).

Le développement ces ports est aussi lié à la loi $n^{\circ} 23.018$ de 1983 , qui met en place des aides financières aux exportations pour les produits miniers, agricoles et halieutiques partant des ports du sud du Río Colorado. Le but est d'améliorer la compétitivité économique de ces régions périphériques du sud de l'Argentine, en faisant baisser le coût des exportations à travers des remboursements des frais de transport et de stabiliser des populations en créant de l'emploi.

Des spécialisations régionales vont se construire, selon les espèces, les bateaux et méthodes de pêche utilisées. Ainsi, dans le Golfo San Matias, on trouve des flottilles diversifiées avec des chalutiers et des

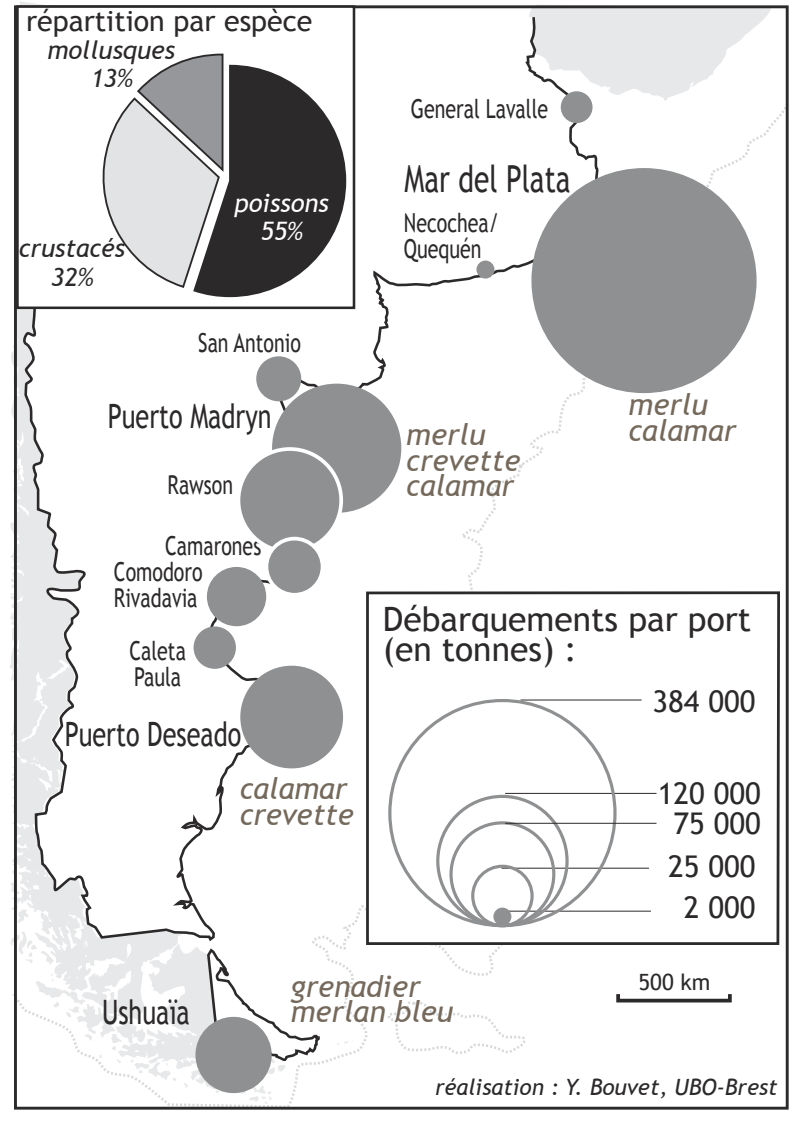

Figure 7 : Les ports de pêche maritime argentins en 2017 (source : SAGyP, 2018)

Argentina fishing ports in 2017

palangriers, souvent artisanaux, débarquant dans les principaux ports comme San Antonio Oeste y Este des produits frais, générant une activité de transformation, de conditionnement et d'expédition vers Buenos Aires et Montevideo.

Plus au sud, les ports voisins du Golfo San Jorge possèdent une flottille de chalutiers hauturiers qui pêchent tant dans les eaux du large que dans les eaux provinciales, venant en concurrence avec la flottille côtière et artisanale. Ils débarquent des produits frais et congelés à Puerto Madryn, Rawson et Comodoro Rivadavia (province du Chubut), et aussi à Caleta Paula et Puerto Deseado (province de Santa Cruz).

Quant aux poteros, ces grands palangriers qui pêchent et congèlent le calamar, s'ils débarquent dans presque tous les ports, on les retrouve principalement à Puerto Deseado et à Ushuaia (province de la Terre de Feu), côtoyant les chalutiers-usines qui traquent le merlu et le grenadier des eaux froides de 
l'extrême sud de la Mer argentine. Ushuaia est aussi le seul port à posséder une flottille de chalutiersusines fabricant du surimi à partir du merlan bleu.

Renforcée par les aides à l'exportation, la forte intégration verticale de la filière pêche en Argentine fragilise le développement des territoires du sud du pays. En effet, de l'armement des navires jusqu'à la vente des produits pêchés, ce sont quelques entreprises, qui achètent et entretiennent les navires, investissent dans les usines de transformation, voire construisent des infrastructures portuaires. En créant de l'emploi et de l'activité économique, elles peuvent négocier un CICT.

Selon le SAGyP, il y a en Argentine une centaine d'entreprises qui produisent, transforment et commercialisent les produits de la mer, dont les dix plus importantes concentrent plus de la moitié des exportations. Ces entreprises, qui peuvent être à forts capitaux étrangers (européens, asiatiques), insufflent l'activité en localisant leurs flottilles et leurs usines, en employant les équipages et les ouvriers des usines, dans un port ou dans un autre selon l'évolution de la productivité d'une pêcherie et l'obtention de CITC, le coût de l'énergie, du transport, de la main-d'œuvre, le cours international des produits de la mer et de la demande intérieure et extérieure. Ainsi, malgré les éléments mis en avant par la loi sur la pêche, des entreprises étrangères captent des CITC pour approvisionner le marché international en utilisant de la ressource maritime argentine.

On voit des fluctuations dans les productions d'une province à l'autre, illustrant la migration d'une flottille vers une pêcherie, l'ouverture ou la fermeture d'une usine, l'investissement dans un port plutôt qu'un autre (voir figure 8). C'est ainsi qu'en complément de Puerto Deseado, pourtant doté d'usines de transformation et de services de réparation navale, mais situé à plus de $100 \mathrm{~km}$ de la route $\mathrm{n}^{\circ} 3$, seule route desservant les villes portuaires de Patagonie, et à $1750 \mathrm{~km}$ de Buenos Aires, s'est créé le port de Caleta Paula, à $200 \mathrm{~km}$ au nord, qui peut accueillir les flottilles de la Province de Santa Cruz et surtout des cargos pour exporter merlus, crevettes et calamars. Cette création s'est faite au détriment du port de Comodoro Rivadavia (port minéralier de la province du Chubut), et au bénéfice également de Puerto Madryn, riche d'entreprises de transformation et qui reçoit, par la route $\mathrm{n}^{\circ} 3$, la production débarquée à plus de $400 \mathrm{~km}$ au sud.



Figure 8 : Évolution des débarquements par province de 2001 à 2017 (source : Secretaria Agricultura Ganadería y Pesca, SAGyP, 2016) Fish landings by Argentina's province 2001-2017

On distingue aussi une diminution des débarquements en Patagonie, liée à la baisse des aides à l'exportation, qui ont disparu en 2016. Pour répondre à la pression de l'Organisation mondiale du commerce, le gouvernement argentin s'est engagé à les faire disparaître en prétextant le coût fiscal démesuré de cette aide (Décret 1199/2016 ${ }^{11}$ ), ce qui suscite de vives discussions entre les provinces de la Patagonie et le gouvernement fédéral, les entreprises halieutiques annonçant la prochaine suppression de 5000 emplois ${ }^{12}$. L'activité, notamment des naviresusines transnationaux, pourraient se reporter vers Mar del Plata.

\section{Mar del Plata, port structurant}

Plus connue comme la grande station balnéaire argentine, Mar del Plata est le centre névralgique de la pêche en Argentine. Ce port accueille tous les types de navires de pêche (au moins 350 bateaux selon les informations du consortium gérant le port de Mar del Plata), du bateau semi-rigide de pêche artisanale au chalutier-usine, débarquant toute sorte de produits, frais, congelés, transformés ou non. Il concentre plus de $50 \%$ des débarquements de pêche

11. Decreto de necesidad y urgencia 1199/2016, [http://servicios.infoleg.gob. ar/infolegInternet/anexos/265000-269999/268508/].

12. El sureño, 29 febrero 2016, [http://www.surenio.com.ar/2016/02/lospuertos-patagonicos-buscan-recuperar-competitividad-frente-a-los-debuenos-aires]. 
maritime d'Argentine, en baisse durant la décennie 1990 durant laquelle 8 tonnes sur 10 y étaient alors débarquées. Malgré le transfert des navires usinescongélateurs vers les ports de Patagonie, Mar del Plata conserve sa suprématie avec plus de $90 \%$ des débarquements de poisson frais, produits qui y sont transformés et commercialisés tant sur le marché intérieur que vers le marché d'exportation. C'est le premier port de débarquement et de transformation du merlu (80\% en frais comme en congelé).

Les navires côtiers et artisans dépendent pour la vente de leurs pêches des groupes industriels (tel que Valastro, Solimeno, Moscuzza...), firmes nationales ou mixtes intégrant l'armement à la pêche, la transformation dans des usines, les espaces de stockage frigorifiques, ainsi que la commercialisation (tant pour l'exportation que vers les chaînes de distribution intérieure, notamment les super et hypermarchés). Ce sont ainsi une centaine d'usines de transformation (filetage, panure et conserves) qui sont implantées à Mar del Plata, essentiellement dans le quartier à l'ouest du port, d'où partent de nombreux camions frigorifiques pour rejoindre, $400 \mathrm{~km}$ plus au nord, le port de Buenos Aires point de départ des principales exportations. Des chantiers de construction et de réparation navales animent la vie portuaire avec des migrations de navires opérant en Atlantique sud qui viennent procéder à l'entretien régulier des navires. C'est aussi à Mar del Plata qu'est installé le principal centre de recherches de l'INIDEP et l'École nationale de pêche. Bien que l'ensemble du port de pêche soit sous gestion privée, la "banquina de pescadores » est le seul lieu accessible au public, permettant d'approcher l'activité halieutique. C'est un site touristique où on peut voir non seulement les bateaux côtiers débarquer leurs poissons (variado costero bonaerense), mais aussi les lobos marinos (lions de mer, symbole de Mar del Plata) évoluer dans le port ou sur les quais; on peut accéder à des magasins de vente directe de poissons frais, ou de conserves qui voisinent avec les magasins de souvenirs et se restaurer dans les petits kiosques pour déguster poissons et fruits de mer. C'est un lieu important du tourisme marplatanense.

Les entreprises halio-alimentaires, par analogie avec l'agro-alimentaire, souvent transnationales, font ou défont l'activité des ports, déplaçant des flottilles sans que les gouvernements provinciaux ou fédéral n'aient de véritables pouvoirs sur l'activité halieu- tique, que ce soit en Patagonie ou dans la province de Buenos Aires (Ramousse, 2009). Pourtant, ce modèle de développement est nocif pour les territoires littoraux puisqu'il entraîne une surexploitation de la ressource, une détérioration de la qualité de vie (par une division extrême du travail à un niveau national et international) qui fragilise les sociétés locales, et provoque une fuite de la rente dégagée par l'exploitation (Svampa, 2011).

\section{Une difficile gestion de la ressource}

La mise en place de quotas ne concerne que les espèces les plus commercialisées. Si cela va dans le sens d'une durabilité de l'activité, cela ne résout pas la pression exercée sur les autres espèces et la concurrence pour l'appropriation de la ressource. Régulièrement, la presse argentine dénonce les actes de pêche illégale réalisés dans les eaux argentines par des navires étrangers (taïwanais ou coréens), ayant acquis des droits de pêche en Uruguay ou aux Malouines. Elle met moins en avant les activités de chalutiers industriels dans les zones côtières, les dépassements de quotas, ou de taille minimale de poisson réalisés par les pêcheurs argentins. Pourtant, c'est le respect des droits de chaque utilisateur de l'espace marin qui permet une durabilité de l'activité halieutique, sinon « l'effet domino » s'applique, les navires de la pêche industrielle irraisonnée des eaux lointaines poussent les navires de pêche hauturière vers les zones côtières qui écrasent la pêche côtière et artisanale (voir figure 9).

Surveiller et protéger, c'est mettre en œuvre un suivi indépendant, public et publicisé de tous les bateaux et de leurs productions, ce qui demande des moyens conséquents, que n'ont pas pour l'instant ni la Préfecture navale, ni le SENASA ou tout autre organisme. Cela demande aussi une indépendance des chercheurs de l'INIDEP, organisme qui dépend encore fortement du pouvoir exécutif fédéral pour ses financements et qui peut subir des pressions pour aménager les quotas. La création en 2014 du projet Pampa azul vient donner une nouvelle place à la recherche sur les espaces marins en Argentine. Ce programme gouvernemental, créé par la Présidente Kichner, et porté par le ministère des Sciences, Technologies et de l'Innovation productive, vise à rassembler et augmenter les moyens de recherches sur l'Atlantique sud pour améliorer les 
connaissances et les technologies d'exploitation des ressources marines, vivantes et minérales. Il vise à renforcer aussi l'activité des industries liées à la mer et à créer une prise de conscience des potentiels maritimes pour la population argentine. On y trouve les organismes publics de recherche, les universités, et certains ministères (agro-industrie, relations extérieures, défense, tourisme, sécurité, environnement et développement durable). Ce très jeune programme n'en est qu'à ses débuts, et déjà la vision industrielle voisine avec celle de la durabilité ${ }^{13}$.

Parallèlement à la connaissance des ressources, le développement d'un circuit de distribution intérieure de produits de la mer de qualité, notamment en frais, crée de la valeur ajoutée à la production de la pêche côtière et artisanale, tout en proposant une alternative nationale à la consommation de la viande pour la population. Les acteurs de la pêche côtière et artisanale cherchent à valoriser leurs productions, sans passer par les grandes entreprises halio-alimentaires, pour assurer leur activité dans les territoires littoraux. Si on peut penser que l'attribution de CITC pour quinze années (depuis 2010) aux entreprises halio-alimentaires assure une durabilité écologique de l'activité, elle ne peut à elle seule soutenir le développement économique, social et territorial des régions littorales de l'Argentine, Ainsi, en Patagonie, la privatisation des richesses marines et des ports de débarquement construit des enclaves exportatrices (Svampa, 2011). Cette privatisation des ressources naturelles crée des droits d'antériorité pour des entreprises, qui seront toujours en recherche de croissance alors que la ressource est épuisable. Cela pose des questions sur la stratégie portée par l'État argentin.

La gestion des pêcheries est encore récente, elle ne concerne que les espèces les plus exploitées et donc les entreprises industrielles de pêche, aucun quota n'est attribué aux pêcheurs artisans. Cette gestion repose sur la qualité des prévisions biologiques notamment pour la définition du total admissible de captures alors qu'une partie du financement de l'INIDEP vient d'un prélèvement sur les exportations de produits marins. La gestion dépend aussi de la surveillance des captures et donc des moyens attribués pour surveiller ce vaste espace maritime fort convoité qu'est la Mer argentine.

13. [http://www.pampazul.gob.ar/iniciativa/].

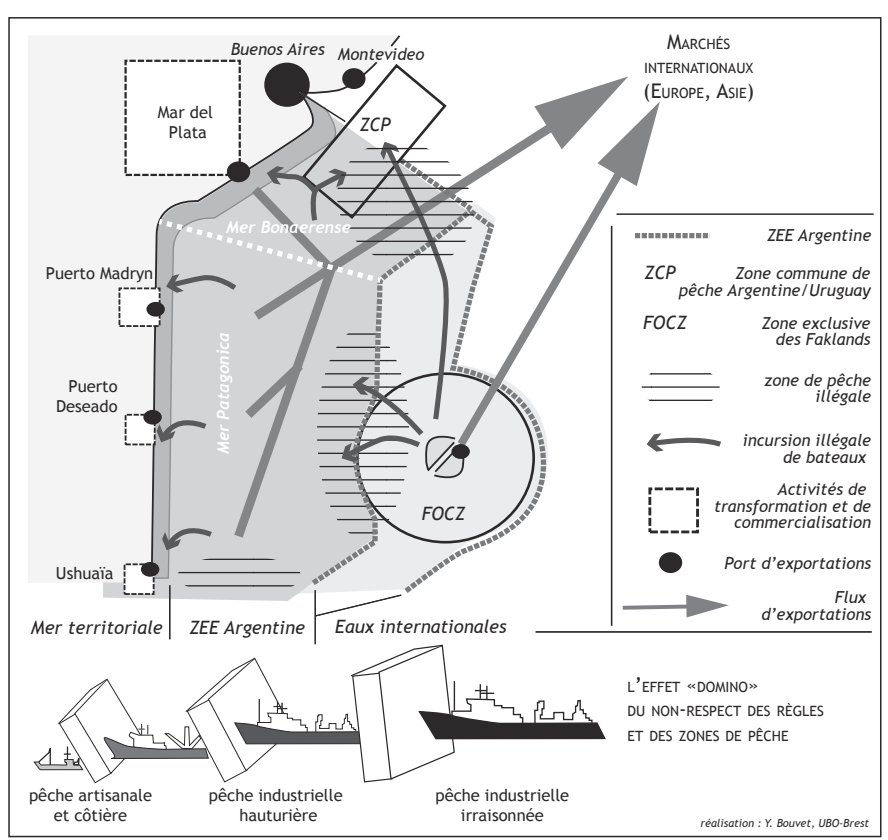

Figure 9 : L'espace halieutique argentin et l'effet domino Argentina fisheries landind and domino effect

En privilégiant une économie de rente d'exportation, l'État argentin ne valorise pas la structuration économique, sociale que l'activité halieutique pourrait avoir sur les territoires littoraux, notamment périphériques. Il minimise aussi le rôle que peut jouer la pêche artisanale sur ces territoires et le soutien à une filière alimentaire de proximité pour la population argentine.

La Ley federal de pesca ne dit pas comment sont redistribués les bénéfices tirés de l'exploitation des ressources naturelles. L'ouverture de la Mer argentine à des acteurs étrangers a pourtant été évoqué en septembre 2016 lors d'un G20 à Hangzou, par le président Macri, qui a offert au président Xi Jinping de faciliter les investissements chinois pour l'exploitation du pétrole et des ressources marines profondes, mettant en avant l'extension de la ZEE argentine ${ }^{14}$.

Au-delà de la gestion biologique de la ressource, la question soulevée ici est celle de la politique de développement des territoires et sociétés littorales argentines. Comment assurer une intégration et une reconnaissance des populations qui vivent, travaillent et valorisent les richesses naturelles marines dans des territoires périphériques? La dis-

14. La Nacion, 4 de septiembre 2016, [www.lanacion.com.ar/1934504-xijinping-mostro-interes-por-el-petroleo-y-el-mar-argentino]. 
parition des aides à l'exportation pour les provinces de Patagonie, en ralentissant l'activité industrielle, peut conduire à une reterritorialisation des mers côtières et aider à construire une autre filière de production et distribution des produits de la mer en mobilisant les acteurs halieutiques (entreprises, pêcheurs artisans, ouvriers d'usines, habitants), à toutes les échelles de réflexion (État fédéral, province, aire urbaine, quartier...).

Cet autre processus de gestion et de répartition des richesses dans une société pluraliste et démocratique est l'unique garant d'un développement équitable et soutenable à long terme. Bien difficile à bâtir dans le contexte actuel, il doit également prendre en compte les voisins (Uruguay, Brésil, Falkland), et bâtir une politique conjointe pour l'Atlantique sud et l'Antarctique pour éviter l'isolationnisme (Sili, 2005, p. 19-25). La reconnaissance possible par l'ONU de la souveraineté argentine sur l'ensemble de la plateforme continentale, donne à l'Argentine le rôle majeur de la gestion durable des ressources naturelles vivantes de l'Atlantique sud.

\section{Conclusion}

Malgré un discours prônant le développement durable, relayé par des lois fédérales, voire provinciales, l'Argentine ne parvient pas à rompre avec les cycles économiques spéculatifs de l'exploitation de son sous-sol (minerais et hydrocarbures), de son sol (soja) ou de sa mer (merlu et crevette). La forte demande qui s'exerce sur ces richesses est au cœur des relations géopolitiques et économiques internationales avec les grandes puissances (notamment la Chine et l'Europe), dans un contexte de fort endettement. L'Argentine, comme nombre de pays, est dans une dépendance aux marchés internationaux, tant pour exporter ses productions que pour financer sa dette. La tentation d'utiliser à outrance les ressources naturelles en ouvrant leur exploitation aux firmes transnationales privées est une vision à court terme d'utilisation du bien commun pour une possible résolution d'un problème économique structurel et mondial. La pêche maritime ne fait pas exception, si ce n'est que les ressources naturelles peuvent être renouvelables, à condition de bien les gérer et de ne prélever que la rente (Bouvet, 2014). Mais la pêche connait une intégration verticale forte de firmes transnationales halio-alimentaires, maîtrisant les investissements, la production, la transformation et la commercialisation. Cela conforte une activité industrielle exportatrice et fournisseuse immédiate de devises, et éloigne la mise en œuvre d'une politique raisonnée, privilégiant la biodiversité et un développement social et économique équitable et équilibré des territoires. Si les ports de Patagonie sont des acteurs de la filière nécessaires au processus exportateur, ils restent dominés par Mar del Plata qui offre une production diversifiée, qui transforme et qui vend autant vers l'extérieur que vers le marché intérieur. À cette vision exportatrice et concentrée des activités, où la concurrence économique et régionale règne, il faudrait privilégier une complémentarité des espaces, tant en mer qu’à terre, pour faire de l'activité halieutique un facteur de développement endogène du territoire argentin.

Au fil de l'histoire des pêches maritimes argentines, le politique et les scientifiques se sont rejoints pour envisager la mer comme un patrimoine commun, avec la création de l'INIDEP, puis avec l'actuel programme de Pampa azul. Cependant l'idée de l'extractivisme continue de dominer, portée par les modèles minier et du soja, et ce malgré la surexploitation des années 1990 sous la pression des flottilles européennes. Si l'exportation de ces produits ont permis de financier des programmes sociaux sous les présidences Kirchner, les bénéfices des exportations de produits de la mer ne bénéficient guère aux territoires littoraux.

L'appropriation des ressources naturelles, à travers la notion de bien commun, est à construire avec un modèle de développement, plus endogène, partant du local, construisant une gestion collective et concertée des ressources marines, portée par l'ensemble des acteurs et accompagné par l'État fédéral et les provinces, et aussi en responsabilisant les consommateurs du Nord. Ce nouveau modèle politique doit réfléchir à plusieurs échelles territoriales et aussi de temporalité, s’il veut être durable, soutenable et partagé, voire démocratique. 


\section{Bibliographie}

Bertolotti M.I., 2008., Pesca responsable y planificación estratégica de la investigación pesquera, in XIII Congreso internacional del CLAD sobre la Reforma del Estado y de la Administración Publica, Buenos Aires, 4-7 nov., 12 p.

Bouvet Y., 2014. De la mer à l'assiette : présentation de la filière halieutique dans le Monde, Géoconfluences, Lyon, 30 p., juillet 2014, [http://geoconfluences.ens-lyon.fr/informations-scientifiques/dossiers-thematiques/oceans-et-mondialisation/corpus-documentaire/de-la-mer-a-12019assiettepresentation-de-la-filiere-halieutique-dans-le-monde].

Campagna C., Verona C., Falabella V., 2005. Situación ambiental en la ecorregión del mar argentino, [http://www. fvsa.org.ar/situacionambiental/Mar\%20Argentino.pdf].

Cañete V., 2008. Las políticas públicas hacia el sector pesquero en la República Argentina entre 1997-2007, in Revista de Estudios Maritimos y Sociales, $\mathrm{n}^{\circ}$ 1, novembre, p. 131-135.

Coccaro J., Le Bail J., Gomez O., Boetto A., 2001. La minería pesquera argentina?, in Reflexiones geográficas, Universidad Nacional de Río Cuarto, nº 10, p. 25-39.

Comisión Nacional del límite exterior de la Plataforma continental Argentina (2016). [http://www.plataformaargentina. gov.ar/].

Falkland Islands Government, 2015. Fisheries Department Fisheries Statistics, Volume 20, 94 p.

Hobert M. et al., 2010. La pesca comercial en la Argentina, Observatorio de políticas públicas, Buenos Aires, 87 p.
Le Bail J., 1998, Conflit et coopération dans l'Atlantique du Sud-Ouest : l'exemple de la gestion des ressources biologiques de la Zone Economique Exclusive argentine, Norois, volume 180, p. 703-714.

Navarro G., Rozycki V., Monsalvo M., 20I4. Estadisticas de la pesca marina en la Argentina. Evolucion de los desembarques 2008-2013, Ministerio de Agricultura, Ganaderia y Pesca de la Nacion, Buenos Aires, 144 p.

Piola A., Rivas A., 1997. Corrientes en la Plataforma continental, in INIDEP. El Mar argentino y sus recursos pesqueros, 1: 119-132 (1997).

Ramousse D., 2009. Des pôles de croissance au développement durable, vers de nouvelles formes de gestion des ressources naturelles dans les espaces périphériques latino-américains?, Cahiers des Amériques latines, n 54-55, p. 15-29.

Sánchez R., Navarro G., Rozycki V., 2012. Estadisticas de la pesca maritima en la Argentina, Evolucion de los desembarques 1898-2010, Ministerio de Agricultura, Ganaderia y Pesca de la Nacion, Buenos Aires, 528 p.

SESAR G.E., 2015. Estudio de mercado de la cadena de suministro del pescado blanco proveniente de la República Argentina. Fundación Vida silvestre, Buenos Aires, 108 p.

SILi M., 2005. La Terre de Feu face à l'avenir. De la crise de territoire à la construction d'un mythe de développement, L'Espace géographie, 2005/1 (tome 34), p. 17-27.

Svampa M., 2011. Néo-« développement » extractiviste, gouvernements et mouvements sociaux en Amérique latine, Problèmes d'Amérique latine, 201 1/3, no 81, p. 101-127. 